\title{
Clinical-diagnostic landmarks and psychiatric comorbidities in borderline and antisocial personality disorders
}

\author{
Ilinca Untu, Alexandra Boloş, Dania Andreea Radu, \\ Roxana Chiriţă, Vasile Chiriță
}

Ilinca Untu - M.D., Ph.D. Student, UMF “Grigore T. Popa”, Iași, “Socola” Institute of Psychiatry, Iaşi, Romania

Alexandra Boloș - M.D., Ph.D., UMF “Grigore T. Popa”, Iași

Dania Andreea Radu - M.D., Ph.D. Student, UMF "Grigore T. Popa”, Iași, "Socola” Institute of Psychiatry, Iaşi, Romania

Roxana Chiriță - Professor, M.D., Ph.D., UMF “Grigore T. Popa", Iași, "Socola" Institute of Psychiatry, Iaşi, Romania

Vasile Chiriță - Professor, M.D., Ph.D., Honorary Member of the Academy of Medical Sciences

\begin{abstract}
Personality disorders in cluster B, borderline and antisocial, which find their counterpart in emotionally unstable and dissocial personality disorder in ICD 10 are some of the most common diagnostic entities in current psychiatric practice, raising a number of clinical issues, regarding characteristic symptomatic elements and especially, frequently associated psychiatric comorbidities, among which are the addictions, mostly the chronic alcoholism and the affective disorders. The present study aims to obtain a synthetic image of the emergency presentation pattern of these cases, their socio-familial background and aggravating psychiatric comorbidities, in a prestigious single-profile medical unit, in order to mark a point of departure for specialized intervention programs.
\end{abstract}

\section{KEYWORDS:}

Emotionally unstable personality disorder, dissocial personality disorder, psychiatric comorbidities.

\section{INTRODUCTION}

Personality is the most complex anthropological structure through which individual identity is defined. In the conditions of a deviant personogenesis, maladaptive features appear, defining the 
ндндн

pathological personalities (1). Their diagnosis is always an approach of a particular complexity because the pathological features can be found in normal people, they manifest differently from one individual to another and from one culture to another, have different degrees of severity and are often associated with episodes or axis I disorders $(2,3)$. The latest psychiatric nosographic systems DSM 5 and ICD 11 approach the diagnosis of pathological personalities differently, coaling categorical and dimensional perspectives (1, 4). The corresponding maladaptive behavior must last at least two years, and the description of each dimension is made only in relation to that of a light, moderate, severe personopathic structure or with a personality with accentuated features $(3,5)$. A conceptual reshaping of personality disorders is being attempted on the territory between the subjective discomfort of the Ego, the relational deficit and the interferences with the dynamics of the other psychiatric sufferings $(1,6)$.

A personality disorder is not only represented by certain isolated areas of individual behavior, it also affects a person's general psychological functioning, interpersonal relationships and social adjustment. These personality disorders are of major importance because of all the implications they have for the diagnosed patients, the families they come from, as well as for the community and society in which they live $(7,8)$. An essential component of these disorders is stability over time, considering that the rigidity, inflexibility, maladaptability of emotional feelings and behavioral manifestations of patients with severe personality disorders, are mentioned throughout the individual biography and not only in certain interpersonal situations. The association with crime, substance abuse, the need for substance use, the increased need for medical care through frequent suicide attempts or even completed suicides, self or hetero physical aggression, is a specific problem regarding society $(1,2,9)$.

For some disorders, it is possible that a psychiatric comorbidity is at least partially an artifact of overlapping definitions and criteria. Comorbidities are common, especially other personality disorders, suggesting that they are biologically vulnerable. Major depressive disorder, dysthymia and conversion disorder may also coexist. The comorbidities between cluster B personality disorders and affective disorders, impulse control disorders and addictions, respectively, corresponding to high values of extroversion and low values of emotional stability and conscientiousness are well known. In this territory, the state of subjective well-being decreases significantly or is strictly reduced to its hedonistic component. Personality disorders frequently coexist with psychotic, affective and anxiety disorders $\quad(2, \quad 9, \quad 10)$ Comorbid psychopathologies result in a significantly greater functional impairment than individual disorders. Comorbid psychopathologies are associated with a poorer prognosis of mood disorders, higher rates of recurrence and chronic outcome, poorer response to treatment, poor adherence to treatment, and an increased risk of suicidal behavior. For the classification of comorbid disorders, the diagnosis should include two or more independent disorders in the same person. Comorbidity extends to other mental disorders. Especially when severe, personality disorders are associated with one or two other disorders. Sometimes the comorbid disorder is very strong, so it dominates the personality disorder. Personality disorder may explain the recurrence of symptoms of mental disorders. The data suggest that there is a common factor for all mental disorders, but no data are available for personality disorder. Personality 
functioning can be an important general factor, being a predictor of poor treatment outcomes. Personality disorder can contribute to the difficulty of treating other mental disorders $(1,10,11)$.

The prevalence of personality disorder, as a categorical entity, seems to be between 4 and $15 \%$. In general, men are about as likely, if not more likely, to be diagnosed with a personality disorder. The prevalence is also higher in people who contact health services more often. A quarter of patients in primary care and half of those in psychiatric care meet the criteria for the disorder. The highest level of prevalence is found in people in contact with the justice system, with two thirds of those incarcerated meeting the criteria for the disorder $(1,2,6,12)$.

Cluster B brings together disorders that involve dramatic, emotional and unpredictable behavior. This category includes:

- Antisocial personality disorder: pattern of carelessness or violation of the rights of others. A person with antisocial TP will not comply with social norms, will lie or deceive others and will act impulsively.

- Borderline personality disorder: pattern of instability in personal relationships, emotional responses, self-image and impulsivity.

- Histrionic personality disorder: pattern of excessive search for attention and excessive emotions.

- Narcissistic personality disorder: pattern of need for admiration and lack of empathy for others $(1,2)$.

\section{MATERIALS AND METHOD}

The retrospective study performed has the role of analyzing the personality disorders in cluster B and the comorbidities associated with them, with the foreshadowing of a model of presentation in psychiatric emergency of patients with such diagnoses. Thus, the study of the distribution of subjects according to demographic data, symptoms and associated comorbidities was followed. The study is a retrospective analysis of the data, collected from the observation sheets of patients admitted to the Institute of Psychiatry Socola Iasi - for personality disorders in cluster B, between January 2018 and January 2019.

Thus, 200 patients were included, male only, in order to maintain a homogeneous group, which is not influenced by the gender variable. In order to obtain the indicators presented in the paper, we performed a systematization of the patients' data, with the establishment of some correlations between the variables taken into account. In order to maintain an appropriate statistical significance and a fairness of the data obtained, we applied as a criterion for fundamental inclusion the diagnosis of emotionally unstable personality disorder and dissocial personality disorder, established according to ICD 10 criteria, mainly to the major addressability of these diagnostic categories in psychiatric emergency, but also to the underdiagnosis of histrionic personality disorder and the absence of individualization of narcissistic personality disorder in the ICD nomenclature, which makes it inapparent in the reported diagnoses of patients included in this research.

The main exclusion criterion was the existence of secondary diagnoses attesting to somatic comorbidities, which could call into question the endogenous origin of the persistent change in personality structure, for the included cases.

\section{RESULTS AND DISCUSSIONS}

Although each personality disorder has its own symptoms, some of them overlap, being common to all personality disorders. The results of the present research showed that 
$67 \%$ of patients have a reduced tolerance to frustration at the time of hospitalization, $70 \%$ have poor impulse control, $69 \%$ of those examined presented with onirophilic aggravation, while $28 \%$ had associated with the consumption of psychoactive substances, $53 \%$ had psycho-motor agitation as a symptom at presentation, and $40 \%$ had depressive symptoms. In addition, $36 \%$ of patients had parasuicidal behavior, while only $15 \%$ had genuine suicidal ideation or attempts. These results reflect only the status at the time hospital admission, which in all cases was performed in a psychiatric emergency, further being exposed the related elements, revealed by the psychiatric interview conducted during the hospitalization in the psychiatric service. The number of selected cases with a diagnosis of emotionally unstable personality disorder and dissocial personality disorder is equal (100 patients for each diagnosis).

Analyzing each of the two types of personality disorders, it was found that in the case of emotionally unstable disorder, most often patients have impulsive manifestations $75 \%$. Psychoemotional lability also occupies a leading place in the symptoms of this disorder, following our study - $67 \%$, followed by reduced tolerance to frustration - $76 \%$. Patients with emotionally unstable personality disorder complain of the feeling of "emptiness" inside - in the case of our study with a frequency of $87 \%$. Following the analysis, we also notice that $53 \%$ have selfharming behavior, $55 \%$ have suicidal behavior, while only $6 \%$ have a paranoid idea. In the case of those with unstable-emotional personality disorder, who present parasuicidal behavior, it is found that $68 \%$ simulate venesection, $20 \%$ control controlled ingestion of drugs and $12 \%$ simulate the attempted hanging. In addition, $50 \%$ of patients with this diagnosis, who present with parasuicidal behavior, also associate with onirophilic aggravation. It is also found that $69 \%$ of patients face an ethanolic addiction syndrome, while $31 \%$ use psychoactive substances. The study also shows that of those addicted to psychoactive substances, $50 \%$ suffer from a co-dependence on alcohol. In $75 \%$ of cases, emotionally unstable personality disorder is added to depressive symptoms, but only in $30 \%$ of cases they meet ICD 10 criteria for a depressive episode. Anxiety spectrum disorders are also among the comorbidities, accounting for $20 \%$, and $15 \%$ of patients have both personality disorder and bipolar disorder.

According to the study conducted within the Socola Institute of Psychiatry, out of the 100 hospitalized patients with dissocial personality disorder, in $70 \%$ of cases, patients present as comorbidity alcohol consumption (ethanolic addiction syndrome), 35\% consume psychoactive substances and $12 \%$ meet the diagnostic criteria for gambling. Of these patients, $45 \%$ have a legal history, most including theft, robbery, rape. In this context, $87 \%$ declare a total indifference to social norms. Among patients diagnosed with dissocial personality disorder, only $15 \%$ have depressive symptoms and 5\% overlap with the diagnostic criteria of a depressive episode. Most of the time, $88 \%$ state that they have impulsive behaviors that lead to heteroagressive and behavioral manifestations.

Among patients with emotionally unstable personality disorder, $79 \%$ have a poor sociofamilial insertion, and among those diagnosed with dissocial personality disorder, $85 \%$. The rest are either employed, but with poor professional stimulation, or have their own families (other than the family of origin), but with frequent conflict situations. 
Of the patients with emotionally unstable personality disorder, $35 \%$ were in the first psychiatric hospitalization, the rest having two or more previous hospitalizations. Of those with dissocial personality disorder, 23\% were at the first hospitalization.

Most patients with dissocial personality disorder were diagnosed between 18 and 25 years of age $(73 \%)$, while for emotionally unstable personality disorder the diagnosis was established especially in the age range 25-35 years $(55 \%)$.

The average length of hospitalization of patients with such a diagnosis is 7 days, shorter in the absence of psychiatric comorbidities or association with addictive pathology and longer when associated with suicidal behavior, parasuicidal or depressive anxiety comorbidities. Regarding the therapeutic conduct, $80 \%$ of those with emotionally unstable personality disorder received thymostabilizers, 50\% received antidepressants, $90 \%$ received benzodiazepines (in their case, however, only $45 \%$ had maintained the recommendation and at discharge, due to the addictive risk of these substances), 56\% received antipsychotics, mostly tiapridum and haloperidol, in order to control the behavioral manifestations. In the case of patients diagnosed with dissocial personality disorder, $90 \%$ received thymostabilizers, $98 \%$ benzodiazepines, with the same mention as for emotionally unstable personality disorder, $68 \%$ received antipsychotics, especially tiapridum, haloperidol or levomepromazine. Out of the total of 200 patients included in the study, only $5 \%$ would have had the financial possibility to address the recommended psychotherapeutic services. However, the main therapeutic intervention required for these patients is psychotherapy, still inaccessible and not firmly established as a method of controlling these mental disorders.

Personality disorders are a special clinical category, through all the implications they have for the diagnosed patients, the families they come from, as well as for the community and society in which they live. Their diagnosis is always an approach of particular complexity because the pathological features can be found in normal people, they manifest differently from one individual to another and from one culture to another, have different degrees of severity and are often associated with others psychiatric pathologies.

This research emphasizes once again the seriousness of the social impact but also the pressure exerted by these two personality disorders on psychiatric medical services, both by their basic manifestations and by the frequent association of psychiatric comorbidities, which themselves represent a real therapeutic challenge.

Suicidal and parasuicidal behavior aggravated by alcohol and / or psychoactive substances, is one of the most common forms of addressability and also a major psychiatric emergency with multiple clinical-therapeutic implications on the management and prognosis of these situations. The results of the study are similar with established data of the specialized literature, outlining a microscale situation that reflects the reality of a prestigious psychiatric institution.

\section{CONCLUSIONS}

Interesting due to their complexity, diversity and the complications they produce, personality disorders represent a strong research motivation, associated with a great desire to clarify and solve them. Personality disorder is a common and chronic mental condition. Its prevalence is estimated 
s between $10 \%$ and $20 \%$ of the general population. People with personality disorders are often labeled as aggravating, demanding or parasitic and are generally considered to have a poor prognosis. About half of all psychiatric patients have a personality disorder, which is a common comorbidity associated with other psychiatric conditions. Personality disorder is also the predisposing factor for other psychiatric disorders (eg, prohibited substance use, parasitic suicidal behavior, affective disorders, impulse control disorders, eating disorders, anxiety disorders) and increases their personal disability, morbidity and mortality. People with personality disorders are more likely to refuse psychiatric help and deny their problems. Comorbidities, as well as pharmacological and / or psychotherapeutic treatment are highlighted as factors that change over time the configuration and intensity of the characteristics of personality disorders.

Emotional and dissocial personality disorder are the most common diagnoses in the personality disorders group that address psychiatric emergency services, both by their symptomatic specificity, and especially by the aggravation caused by a series of psychiatric comorbidities. Knowing the presentation model of these nosological entities can prevent a series of complications and can create a preamble for specific initiatives in the direction of the preventive and therapeutic approach. The risks brought by these two personality disorders include hetero and self-aggression, suicide, decreased social productivity, forensic acts, sometimes with extremely serious impact. Although the frequency of all personality disorders is comparable, these two exponents of cluster B with their correspondent taken into account in the present study, from ICD 10, are most apparent through the major impact of personality traits on self-perception and the surrounding world, through the impulsiveness and unstable background that accompanies them.

\section{ACKNOWLEDGEMENTS AND DISCLOSURES}

The authors state that they are no declared conflicts of interest regarding this paper.

\section{REFERENCES}

1. Kraus, G., Reynolds, D. J. (2001). The "abc's" of the cluster b's: Identifying, understanding, and treating cluster b personality disorders. Clinical Psychology Review, 21(3), 345-373.

2. Davison, S. E. (2002). Principles of managing patients with personality disorder. Advances in Psychiatric Treatment, 8(1), 1-9.

3. Martin S., Del-Monte J., Graziani P. (2019), Impulsivity issues in borderline personality disorder and it's links with insight: the role of urgency, Heliyon, 10.1016/j.heliyon.2019.e02564, 5, 10.

4. Dang Zheng, Jie Chen, Xiaoming Wang, Yuan Zhou (2019), Genetic contribution to the phenotypic correlation between trait impulsivity and resting-state functional connectivity of the amygdala and its subregions, NeuroImage, 10.1016/j.neuroimage.2019.07.010.

5. Kulacaoglu F., Kose S. (2018), Borderline Personality Disorder (BPD): In the Midst of Vulnerability, Chaos, and Awe, Brain Sciences, 10.3390/brainsci8110201, 8, 11, (201).

6. Corniquel M.B., Koenigsberg H.W., Likhtik E., Toward an animal model of borderline personality disorder, Psychopharmacology, 10.1007/s00213-019-05289-x.

7. Wilson S, Stroud CB, Durbin CE. Interpersonal dysfunction in personality disorders: A meta-analytic review. Psychol Bull. 2017;143(7):677-734. doi:10.1037/bul0000101.

8. van Dijk, S., Veenstra, M. S., Bouman, R., Peekel, J., Veenstra, D. H., van Dalen, P. J., van Asselt, A., Boshuisen, M. L., van Alphen, S., van den Brink, R., Oude Voshaar, R. C. (2019). Group schema-focused therapy enriched with psychomotor therapy versus treatment as usual for older adults with cluster B and/or C personality disorders: a randomized trial. BMC psychiatry, 19(1), 26.

9. Koppers, D., Kool, M., Van, H., Driessen, E., Peen, J., Dekker, J. (2019). The effect of comorbid personality disorder on depression outcome after short-term psychotherapy in a randomised clinical trial. BJPsych open, 5(4), e61.

10. Goorden, M., Willemsen, E., Bouwmans-Frijters, C., Busschbach, J., Noomx, M. J., van der Feltz-Cornelis, C. M., Uyl-de Groot, C. A., Hakkaart-van Roijen, L. (2017). Developing a decision tool to identify patients with personality disorders in need of highly specialized care. BMC psychiatry, 17(1), 317. 
Bulletin of Integrative Psychiatry O New Series OJune 2020 ○ Year XXVI ONo. 2 (85)/35

11. Smit, M. L, Fens. D. J. Ban, D. L , 11. Smits, M. L., Feenstra, D. J., Bales, D. L., de Vos, J., Lucas, Z., Verheul, R., Luyten, P. (2017). Subtypes of borderline personality disorder patients: a cluster-analytic approach. Borderline personality disorder and emotion dysregulation, $4,16$.

12. Ekselius L. (2018). Personality disorder: a disease in disguise. Upsala journal of medical sciences, 123(4), 194204.

\section{Correspondence:}

Roxana Chiriță,

Professor, M.D., Ph.D., UMF “Grigore T. Popa”, Iași, “Socola” Institute of Psychiatry, No. 36 Bucium, Iaşi, Romania, d.stigma@gmail.com

Submission: 10 apr 2020

Acceptance: 29 may 2020 